\title{
The Two Micron All-Sky Survey: Removing the Infrared Foreground
}

\author{
John E. Gizis \\ Infrared Processing and Analysis Center, California Institute of \\ Technology, Pasadena, CA 91125 USA \\ Michael F. Skrutskie \\ Dept of Astronomy, University of Massachusetts, Amherst, MA 01003 \\ $U S A$
}

\begin{abstract}
We introduce the properties of the Two Micron All-Sky Survey (2MASS) for IAU Symposium 204. 2MASS is a near-infrared survey of the entire sky characterized by high reliability and completeness. Catalogs and images for $47 \%$ of the sky are now available online. This data release has been used by Wright (2000) and Cambrésy et al. (2000) to subtract the stellar foreground at 1.25 and 2.2 microns from COBE DIRBE data, revealing the cosmological near-infrared background.
\end{abstract}

\section{Introduction}

The Two Micron All-Sky Survey (2MASS) is an ongoing survey to map the entire sky at near-infrared wavelengths. Motivated by the particular application of 2MASS to the determination of the extragalactic near-infrared background, our goal in this paper is to review the status and quality of 2MASS data. The 2MASS dataset has many applications, and it is our hope that this introduction to the data will motivate other investigators to utilize the $47 \%$ of the sky presently publicly available. The 2MASS catalogs and images are available online at http://www.ipac.caltech.edu/2mass/ and are fully described by Cutri et al. (2000).

\section{Overview of 2MASS}

\subsection{Survey Characteristics}

The primary goal of 2MASS is to produce a uniform and unbiased near-infrared survey of the entire sky characterized by high reliability and completeness. It is thus a modern version of the classic Palomar sky survey (POSS) - but with digital, fully calibrated data in a new wavelength regime (Fig. 1). 2MASS is a joint project of the University of Massachusetts and the Infrared Processing and Analysis Center (IPAC)/California Institute of Technology, funded by the National Aeronautics and Space Administration and the National Science Foundation. The primary task of U. Mass has been to fabricate and operate two 1.3-meter telescopes (one at Mt. Hopkins, Arizona, and the other at CTIO, 
Chile) equipped with multi-band infrared cameras. IPAC has developed and maintained a data pipeline capable of routine processing of $20 \mathrm{GBy}$ of raw data per day into source lists and images. The results must be distributed to the scientific community in a timely fashion.

Some of the characteristics of 2MASS are summarized in Table 1. The cameras use beam-splitters to obtain $\mathrm{J}, \mathrm{H}$, and $\mathrm{K}_{s}$ simultaneously. This is an important advantage of 2MASS, since there is no possibility of producing anomalous colors due to variable or transient objects. If an object found in one band is not detected in other bands, upper limits on its brightness are measured.

While uniformity of the survey is a primary goal, varying observing conditions (background and seeing) have effects on the achieved sensitivity. Observations that do not meet the sensitivity requirements in Table 1 are rescanned. The $\mathrm{K}_{s}$ backgrounds are driven by thermal emission - as a result, winter observations at Mt. Hopkins are as much as 0.7 magnitudes deeper than the requirements, imprinting a systematic effect on the sky. The $\mathrm{H}$ backgrounds are dominated by airglow, which is highly variable and not very dependent on season.

\begin{tabular}{lc}
\multicolumn{1}{c}{ Table 1. 2MASS Characteristics } \\
\hline Arrays & $256 \times 256$ NICMOS3 $(\mathrm{HgCdTe})$ \\
Wavebands & $\mathrm{J}, \mathrm{H}$, and $\mathrm{K}_{s}(2.00-2.32 \mu \mathrm{m})$ \\
Telescopes & 1.3 -meter Equatorial Cassegrain \\
Pixel Size & $2.0^{\prime \prime}$ \\
Integration time & $6 \times 1.3 \mathrm{~s} /$ frame $=7.8 \mathrm{~s}$ total \\
Sensitivity $(10 \sigma)$ & $15.8,15.1,14.3$ mag for $\mathrm{J}, \mathrm{H}$, and $\mathrm{K}_{s}$ \\
Photometric Accuracy & $5 \%$ for bright sources $(3 \%$ in practice $)$ \\
Photometric Uniformity & $4 \%$ over the sky $(1 \%$ in practice $)$ \\
Positional Accuracy & $0.5^{\prime \prime}\left(0.2^{\prime \prime}\right.$ in practice $)$ \\
Completeness / Reliability & $0.99 / 0.9995$ \\
\hline \hline
\end{tabular}

The unit of the 2MASS survey is the $6^{\circ} \times 8.5^{\prime}$ tile. Each tile consists of 273 overlapping exposures (Figure 2). As a result, each star is imaged 6 times and total exposure time is $1.3 \mathrm{~s} /$ frame $\times 6=7.8$ seconds. The sky is divided into $\sim 60,000$ tiles, which overlap slightly in right ascension (Figure 3 ). The 6 samples allow bad pixels and cosmic rays to be eliminated, and also $1^{\prime \prime}$ pixels to be generated for the final data products from the $2.0^{\prime \prime}$ camera pixels.

\subsection{Data Products}

The 2MASS data products consist of catalogs of sources and an atlas of images. The Point Source Catalog (PSC) includes $\sim 300$ million sources with astrometric and photometric information (Figure 4). Sources down to signal-to-noise ratios of 7 are included in the PSC. The Extended Source Catalog (XSC) includes $\sim 2$ million objects. Jarrett et al. (2000a) give a full description of the extended source processing. The extended sources are identified on the basis of morphology, and are mostly galaxies - the software has been designed to exclude double stars from the XSC. Because galaxies are typically redder $\left(\mathrm{J}-\mathrm{K}_{s}>1\right)$ than stars, a G-score is also available using color information to improve the reliability of the galaxy catalog. "Snapshot" images of all extended sources are available on- 


\section{POSS 2MASS}

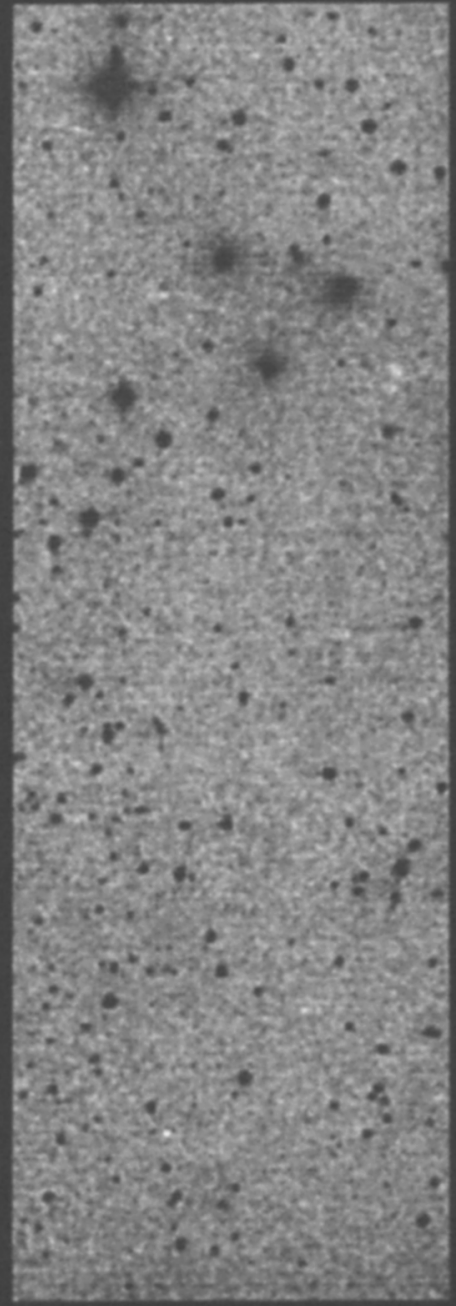

$0.43 \mu \mathrm{m}$

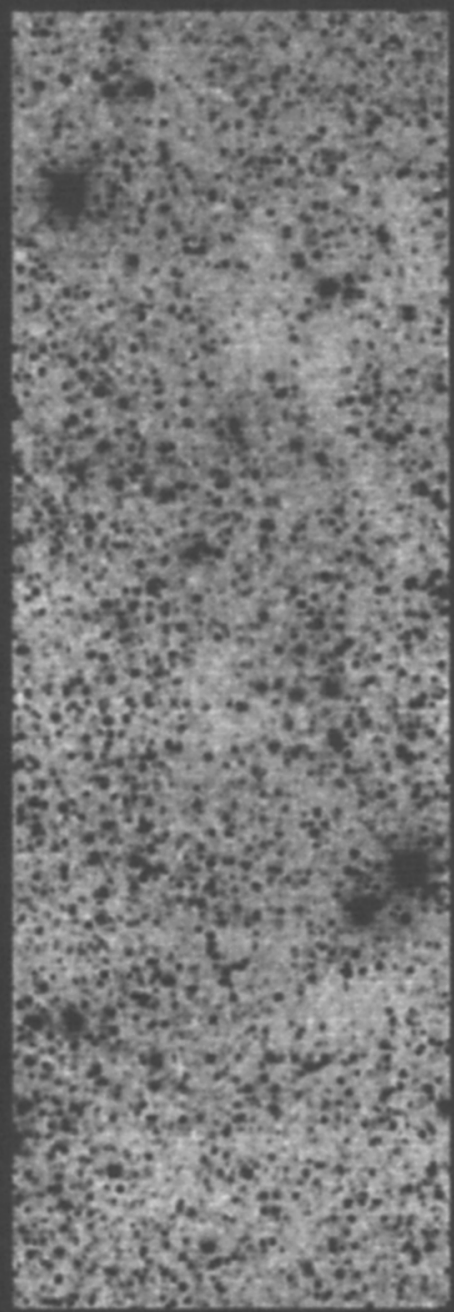

$2.17 \mu \mathrm{m}$

Figure 1. POSS optical observations compared to 2MASS in the Galactic Plane 
Right Ascension

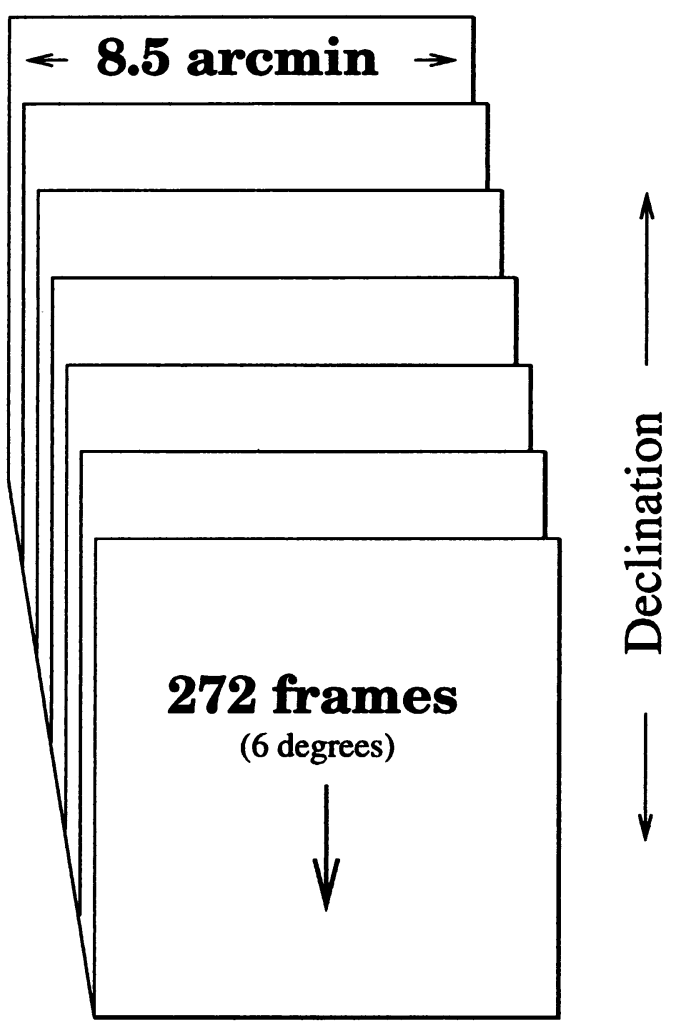

Figure 2. 2MASS Scanning Method: Each point on the sky is imaged 6 times. 


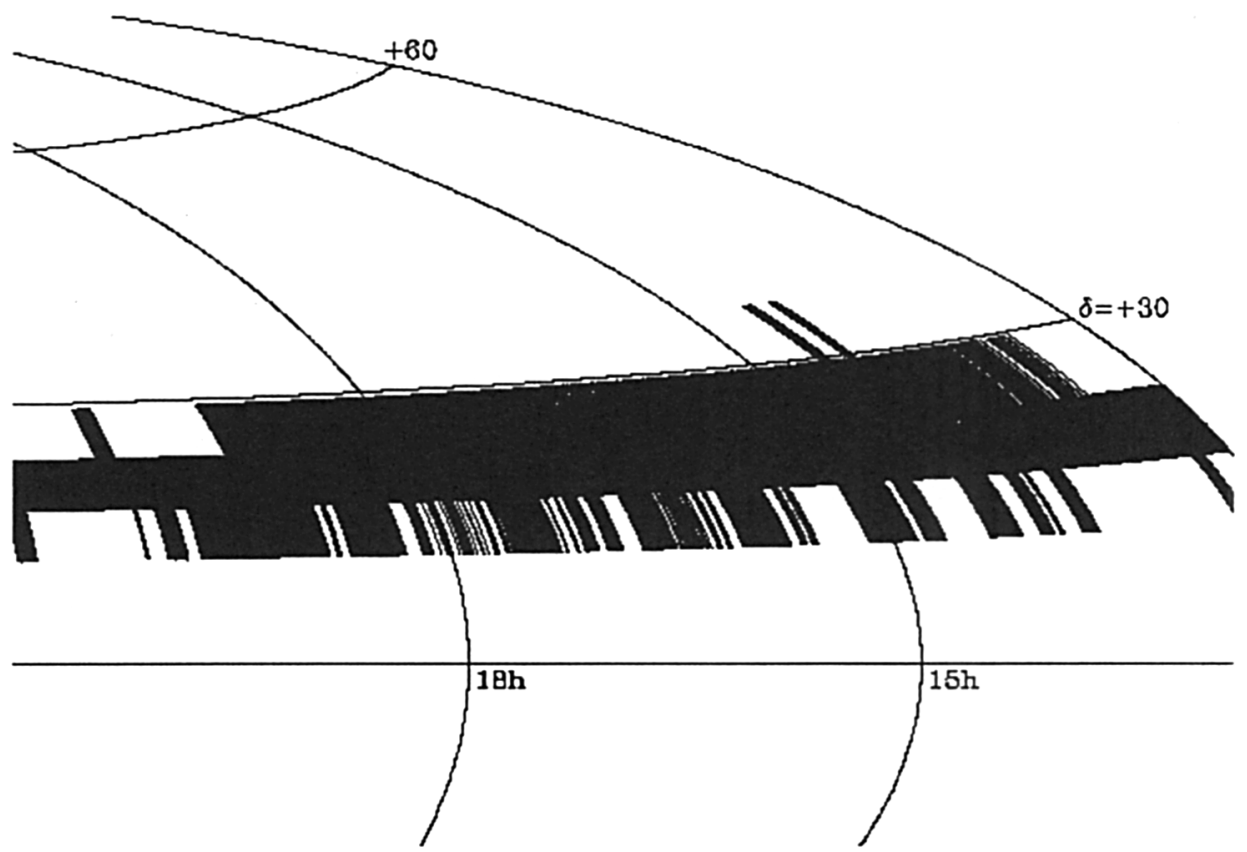

Figure 3. 2MASS Scanning Strategy: The sky is divided into overlapping tiles.

line. It should be noted that the PSC includes many galaxies too faint to be resolved - most can be identified by their 2MASS or 2MASS+optical colors.

The 2MASS Digital Atlas of the sky consists of 1.8 million calibrated $512 \times 1024$ images in the three passbands (11 TBy). Presently, the Quicklook atlas is available - these images have been lossy-compressed. They are useful for finding charts or visually verifying a source, but cannot be used for photometry. The full, uncompressed images in FITS format are expected to be available online soon.

The data products (presently $47 \%$ of the sky) are available online through the Infrared Science Archive (IRSA). IRSA offers the capability to efficiently query the catalog using SQL commands. Source lists may be uploaded to IRSA for positional searches. DVD versions of the catalog releases are also available.

\subsection{Photometric Quality}

Because it is so critical to so many applications - particularly the extragalactic background - the photometric quality of 2MASS deserves additional discussion. Calibration is obtained once an hour by six repeated one-degree survey strips (providing 36 observations of each star). The strips are centered on NICMOS calibrators (Persson et al. 1998). Although initially only the single NICMOS calibrators were available, using the first year and a half of 2MASS data Nikolaev et al. (2000) identified and calibrated $\sim 50$ secondary stars per field. Their global analysis demonstrates that the photometric system is uniform around the 


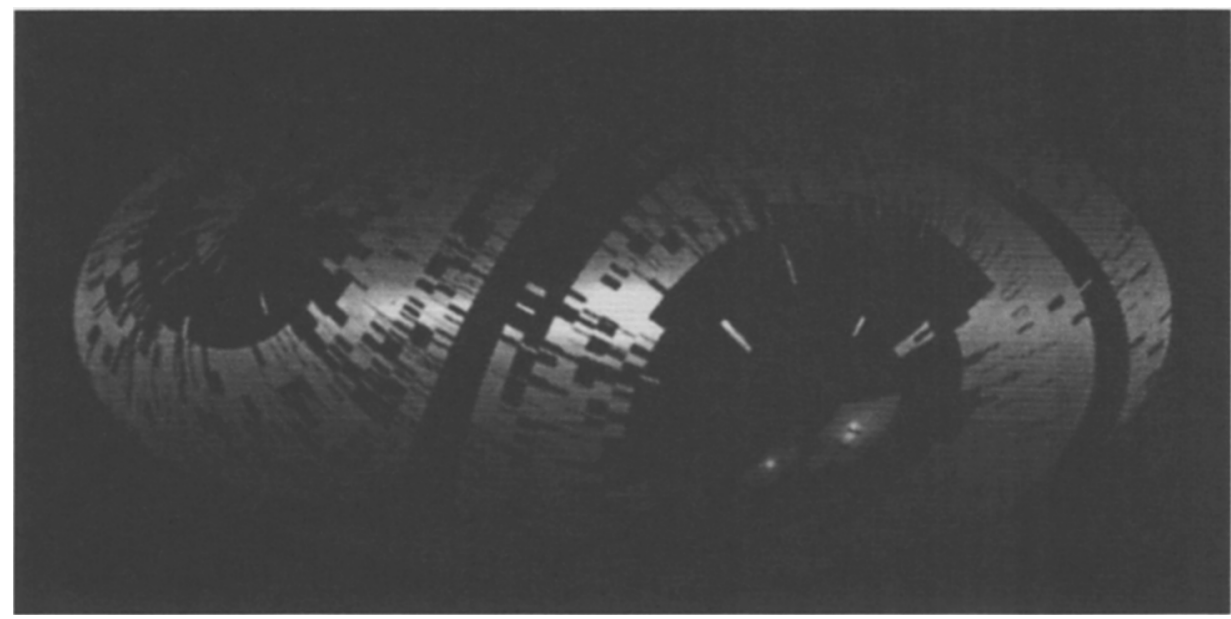

Figure 4. Star Counts for the 2MASS Second Incremental Release.

sky and in time at the $1 \%$ level. There is no evidence for color terms between the two hemispheres. The nightly zeropoints are determined to better than $1 \%$ - an example is shown in Fig. 5. Furthermore, the repeatability of all the high signal-to-noise stars in the strips are examined to verify that conditions are photometric. The RMS statistics of the 6 repeats (Fig. 6) are computed to monitor the sensitivity of the system. The extensive dataset from these observations allow us to quantify the sensitivity in terms of the background and seeing, allowing the quality of the science scans to be assessed. Clouds between calibration observations can be identified by comparing the photometry in science scan overlap regions and by their high and variable $\mathrm{K}_{s}$ emission.

\section{Science Applications}

The near-infrared offers two special advantages over optical observations. First, interstellar dust is more transparent in the IR $\left(A_{K} \approx 0.1 A_{V}\right)$. As a result, the "Zone of Avoidance" caused by the Milky Way is reduced. Furthermore, internal extinction, such as in star formation regions and other galaxies, is minimized. Second, the near-infrared is more sensitive to "cool" objects. This enables the study of IR-selected populations underrepresented in optically-selected samples. For galaxy studies, the older stellar population emits a larger fraction of IR light, giving a better handle on the true mass distribution.

As a taste of the diverse uses of 2MASS data, we briefly mention some recent studies. Within the solar system, Sykes et al. (2000) show that 2MASS data can classify asteroids on the basis of their composition. The census of the local solar neighborhood is being completed by the discovery of very-late $M$ dwarfs (Gizis et al. 2000), L dwarfs (Kirkpatrick et al. 1999), and T Dwarfs (Burgasser et al. 1999) $-\mathrm{L}$ and $\mathrm{T}$ dwarfs are new spectral classes $\left(T_{\text {eff }}<2200 \mathrm{~K}\right)$ of brown dwarfs. Star counts constrain Galactic structure (Ojha, this volume) and show the existence of flaring and warping of the outer Galactic disk (Alard 2000). 

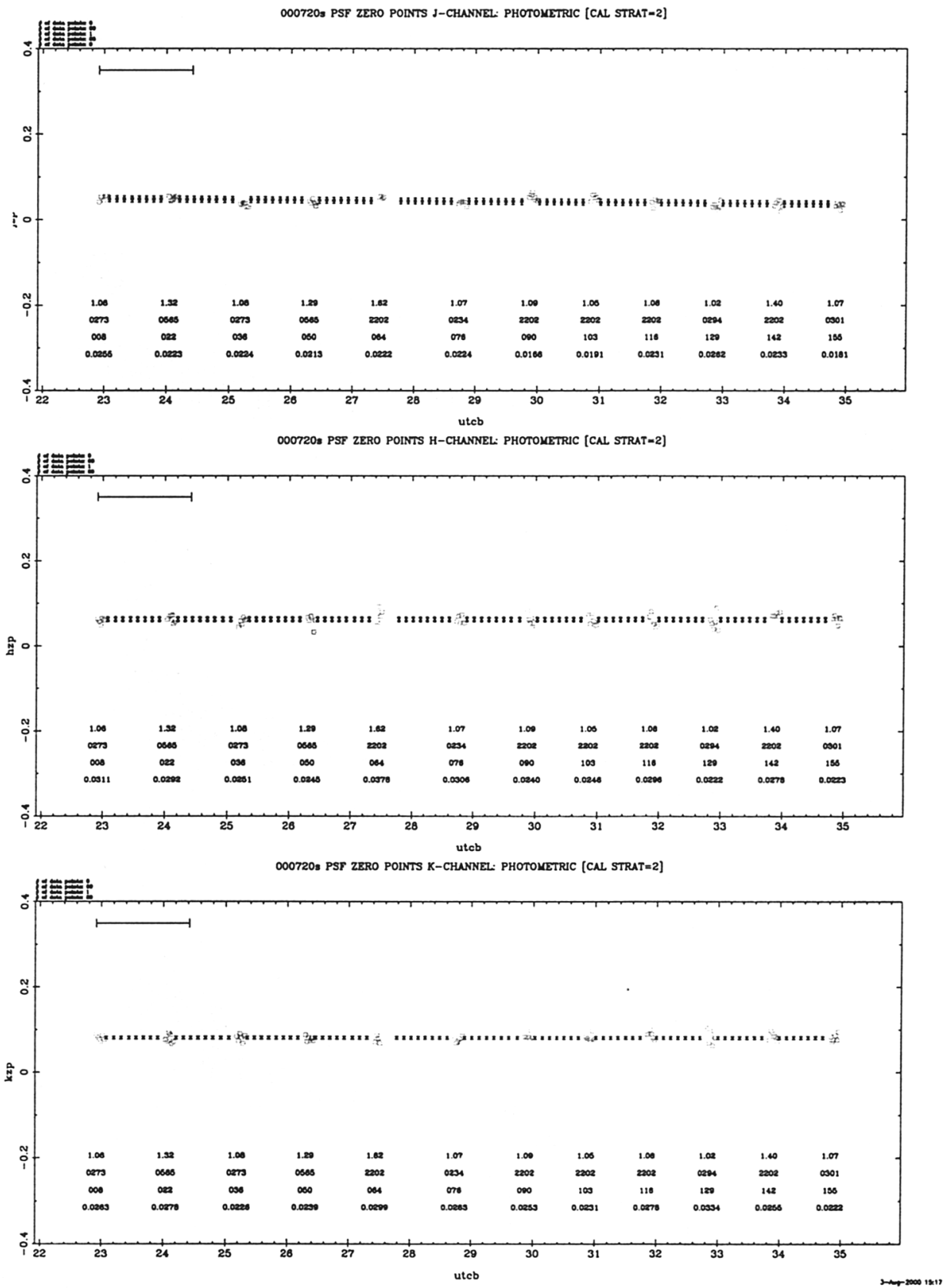

Figure 5. Zero-point solution for 20 July 2000 CTIO observations. 


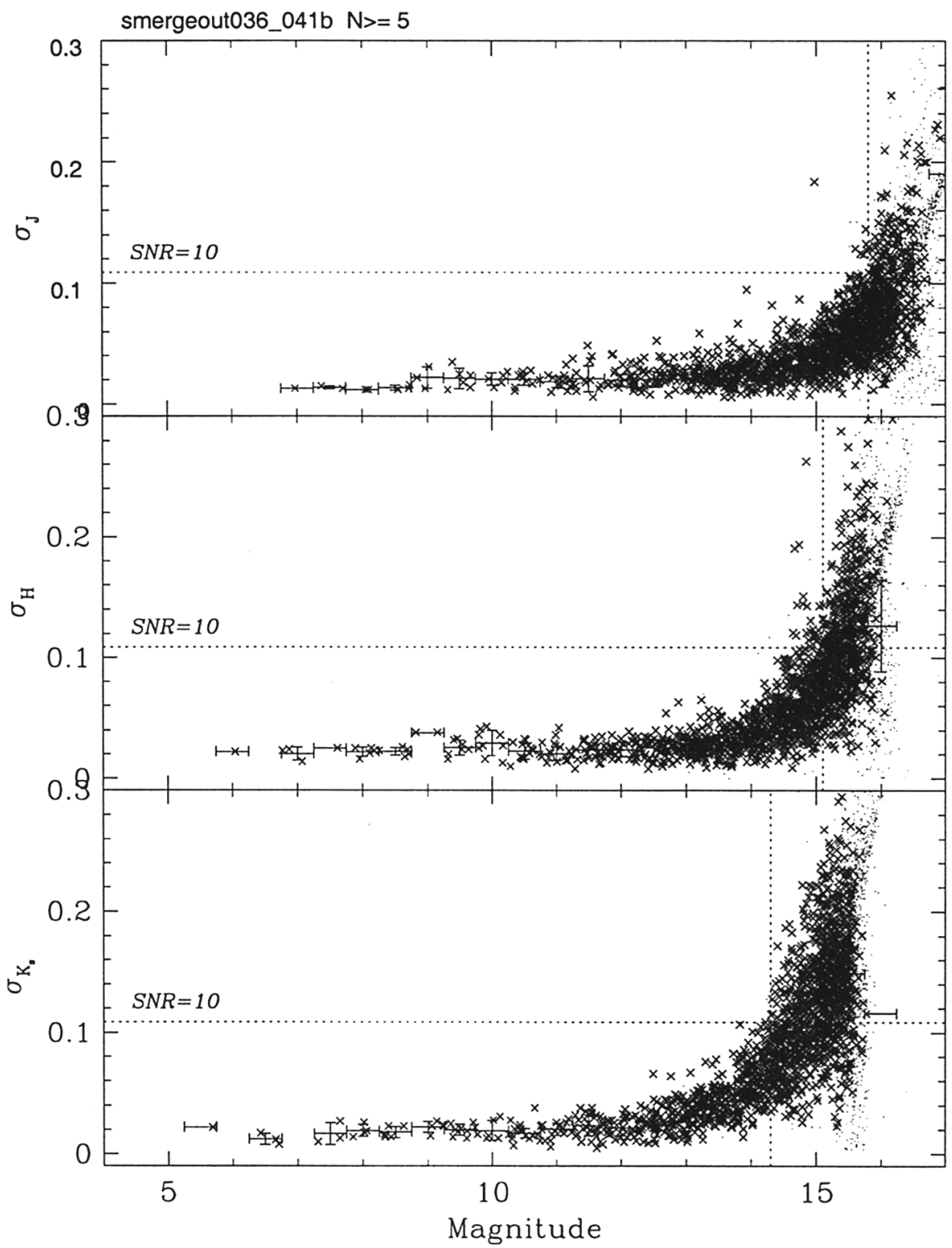

Figure 6. Repeat statistics for one calibrator set on 20 July 2000. 
2MASS is also contributing to extragalactic astronomy. Still within the Local Group, the structure of the Large Magellanic Cloud - and the interpretation of MACHO microlensing results - is discussed by Weinberg \& Nikolaev (2000). Large-scale structure behind the Zone of Avoidance is revealed by Jarrett et al. (2000b). Infrared selection has uncovered a population of very red quasars and AGN (Nelson et al. 1999). Remarkably, 2MASS also is relevant for studies of the cosmological background, discussed in the next section.

\section{The Near-IR Foreground and Extragalactic Background}

The use of 2MASS for determining the cosmological background has been pioneered by Wright (2000), whom we follow in this discussion and whose work the interested reader is urged to consult. The DIRBE experiment on COBE (see Hauser, this volume) provides calibrated observations of the entire sky at 1.25 and 2.2 microns, corresponding to the 2MASS J and $\mathrm{K}_{s}$ filters. The DIRBE beam size is $0.7^{\circ} \times 0.7^{\circ}$ - thus, many stars are included in each DIRBE beam. Arendt et al. (1998) found that the uncertainties and systematic errors in galactic star count models are large enough that a detection of the infrared background at 1.25 and 2.2 microns was not possible. They report that the uncertainty due to stars is $15 \mathrm{nW} \mathrm{m} \mathrm{sr}^{-2} \mathrm{sr}^{-1}$ at 1.25 microns and $10 \mathrm{nW} \mathrm{m} \mathrm{m}^{-2} \mathrm{sr}^{-1}$ at 2.2 microns - to be compared with an estimated uncertainty of 15 and 6 respectively in the zodiacal light model. Since the cosmological background is approximately this magnitude - and since Arendt et al. find systematic residuals as a function of galactic latitude - a more direct approach is needed.

Wright (2000) has approached this problem by using the 2MASS data release to directly estimate the contributions of stars to the observed DIRBE fluxes. (Gorjian, Wright, \& Chary 2000 used their own 2.2 and 3.5 micron observations to analyze a single dark spot.) Selecting DIRBE dark regions minimizes the contribution of zodiacal light. Wright found four DIRBE dark regions with complete 2MASS coverage in the data release - even in these pixels, zodiacal light contributed $\sim 75 \%$ of the observed DIRBE 2.2 micron signal. A further $\sim 15 \%$ is contributed by foreground stars. The remaining signal is due to the desired extragalactic background. (Cambrésy et al. (2000) estimate that the 2MASS-resolved galaxies contribute only $5 \%$ of the light.) Wright has shown that the 2MASS data allows the stellar contribution to be subtracted from the DIRBE data on a pixel-by-pixel basis - there is an excellent correlation between the 2MASS integrated star brightness and DIRBE flux. This allows a detection of the extragalactic background light and for strict limits to be placed on fluctuations in the background. Wright (2000) uses the 'no-zodi principle' zodiacal light models of Wright (1998).

The star-subtraction approach has been extended by Cambrésy et al. (2000), who use the public 2MASS PSC to subtract the stellar contribution over a large area of the sky. This allows them to confirm the isotropy of the putative extragalactic background - a sign that the background is truly cosmological. 2MASS's determination of the stellar contribution is precise enough that the zodiacal modelling dominates the uncertainty, although the contribution of very faint stars and the flux calibration make small contributions (see the discussions throughout this volume). In particular, use of the Kelsall et al. (1998) zodiacal 
light model makes Cambrésy et al.'s estimated cosmological background higher than Wright's (2000) estimates. Wright estimates that $94 \%$ of the uncertainty in the cosmological light is due to the zodiacal light model at both 1.25 micron and 2.2 microns. 2MASS has thus allowed the stellar uncertainty to be reduced from being the dominant term (Arendt et al. 1998) to being a negligible one. For the actual results and interpretation, see Wright (2000) and Cambrésy et al. (2000).

\section{Summary}

Nearly half the sky is now publicly available. At the time of this writing (11 October 2000 ), $99.8 \%$ of the sky has been observed with catalog-quality data. The present data release to the astronomical community has enabled studies from the solar system to cosmology even before the final processing of the full 2MASS dataset.

\section{References}

Alard, C. 2000, MNRAS, submitted (astro-ph/0007013)

Arendt, R. G., et al. 2000, ApJ, 508, 74

Burgasser, A. J., et al. 1999, ApJ, 522, L65

Cambrésy, L. et al. 2000, ApJ, submitted

Cutri, R. et al. 2000, Explanatory Supplement to the 2MASS Second Incremental Data Release, http://www.ipac.caltech.edu/2mass/

Gizis, J. E., et al. 2000, AJ, 120, 1085

Gorjian, V., Wright, E. L., \& Chary, R. R. 2000, ApJ, 536, 550

Jarrett, T. H., et al. 2000a, AJ, 119, 2498

Jarrett, T. H., et al. 2000b, AJ, 120, 298

Kelsall, T. et al. 1998, ApJ, 508, 44

Kirkpatrick, J. D., et al. 1999, ApJ, 519, 802

Nelson, B., et al. 1999, in Astrophysics with Infrared Surveys: A Prelude to SIRTF, (ASP Conf. Ser. 177), ed. M.D. Bicay, R.M. Cutri, \& B.F. Madore, 150

Nikolaev, S., et al. 2000, AJ, 120, 3340

Persson, S. E., et al. 1998, AJ, 116, 247

Sykes, M. V., et al. 2000, Icarus, 146, 161

Weinberg, M. D. \& Nikolaev, S. 2000, ApJ, submitted (astro-ph/0003204)

Wright, E. L., 1998, ApJ, 496, 1

Wright, E. L. 2000, ApJ, submitted (astro-ph/0004192) 


\section{Discussion}

Mike Werner: Are there plans for the telescopes after the completion of the survey, and could they do useful things relevant to the goals of this conference?

John Gizis: There are no current plans for future use of the 2MASS telescopes once Survey operations conclude. The Southern Hemisphere facility will spend as much as three months executing a Magellanic Clouds survey that will use an integration time 6 times longer (about a magnitude deeper) than normal survey integrations. With this sensitivity the AGB and RGB evolutionary "bumps", which are sensitive to metallicity and age, are observable. These data can be reduced using the standard 2MASS pipeline reduction and the project plans to make the results publicly available.

Charley Lineweaver: Have you discovered any new nearby $M$ and $L$ dwarfs?

Gizis: New nearby $M$ and $L$ dwarfs are reported among others in Kirkpatrick et al. (1999)and Gizis et al. (2000) (see references above). 2MASS should allow all nearby $\mathrm{M}$ and $\mathrm{L}$ dwarfs to be identified. 